Gut, 1962, 3, 219

\title{
Fluid and electrolyte disturbances in patients with long-established ileostomies
}

\author{
N. D. GALlAGHER, D. D. HARRISON, AND A. P. SKYRING \\ From the Gastroenterology Unit, Royal Prince Alfred Hospital, Camperdown, N.S.W., Australia
}

EDITORIAL SYNOPSIS Salt and water depletion has been noted in 10 patients with long-established ileostomies. Marked renal conservation of sodium and water was present in a number of apparently healthy ileostomy patients. The sodium and potassium concentrations of ileostomy material showed little variation when these patients were in good health but there was a decrease in the sodium concentration and an increase in the potassium concentration during episodes of salt depletion. Greater attention needs to be paid to the fluid and electrolyte requirements of ileostomy patients after they have left hospital.

Increased fluid and electrolyte losses are the inevitable result of an ileostomy. They are most marked during the post-operative period and continue even when normal ileostomy function is established. Fowler, Cooke, Brooke, and Cox (1959) state that the body is then well able to conserve essential fluid and electrolytes; this view is supported by two large follow-up studies by Rogers, Bargen, and Black (1954) and by Brooke (1956) who do not mention the occurrence of fluid and electrolyte disturbances. However, these disturbances are not uncommon in our experience as 10 of 60 out-patients with ileostomies who were seen in the period January 1959 to June 1961 developed symptoms of sodium and water depletion. With a view to decreasing the frequency of the latter complication we have studied fluid and electrolyte losses both in apparently healthy and in salt-depleted patients. The clinical features of the 10 patients with salt and water depletion and the results of the study will be described.

\section{CLINICAL EXPERIENCE}

Ten patients who had had either a subtotal or total colectomy with ileostomy for ulcerative colitis from one month to two years previously developed sodium and water depletion. Five of the group were admitted to hospital. Each patient had been in good health until the onset of the present illness.

Acute sodium and water depletion occurred in three patients as a result of an illness suggestive of gastro-enteritis. Each patient complained of diarrhoea and vomiting; other members of one patient's family had a similar illness. One patient fainted after his ileostomy bag rapidly filled; another developed postural hypotension and was anuric for two days before admission. A third patient had marked hypotension when seen by the referring doctor and was found to be dehydrated on arrival. Rapid intravenous fluid and electrolyte replacement was necessary in two patients to restore their usual state of health.

Sodium depletion developed less dramatically in seven patients, two of whom became oliguric. Three patients complained of headache, anorexia, nausea, and muscle cramps during a hot spell. Salt and water intake was increased but several days passed before a sense of well-being returned. Another three patients had symptoms of sodium depletion during episodes of transient intestinal obstruction producing colicky abdominal pain and diarrhoea. Anorexia, malaise, and muscle cramps disappeared after crushed salt tablets were taken and the obstruction subsided.

The remaining patient, an 18-year-old boy from an outlying area of the state who had developed a peptic stricture of the duodenum and malabsorption, was admitted with ileostomy obstruction. Large volumes of ileostomy material were lost after the obstruction was relieved and the patient had a generalized convulsion before sodium imbalance was corrected. He was quite well on leaving hospital but one year later was flown to Sydney after being stranded under a hot sun for eight hours without water. He was found to have marked hyponatraemia, oliguria, and azotaemia. Intravenous fluid and salt replacement therapy brought about a rapid improvement. 
Twenty out-patients who had had a procto-colectomy and ileostomy for ulcerative colitis one month to five years previously and who were in apparent good health were studied.

Each patient was provided with polyethylene containers for the collection of 24-hour specimens of urine and ileostomy material. Collections of urine were also obtained from 20 members of the hospital staff and medical students who served as controls. No estimate was made of fluid and electrolyte losses in sweat. Studies were performed in the early winter months to minimize the effects of the Australian summer.

The tops of the polyethylene containers containing the ileostomy material were cut away and a measured volume of glass-distilled water was added. The contents were then homogenized by an overhead blending assembly at 1,000 r.p.m. for at least three minutes. The fluid homogenate was transferred to a measuring cylinder and the total volume measured. The volume of ileostomy material was obtained by difference. An aliquot of well-mixed homogenate was centrifuged at 2,500 r.p.m. for 10 minutes and the aqueous supernatant was analysed for sodium and potassium in a Perkin-Elmer flame photometer. Urine volumes in both groups were measured directly and sodium and potassium contents estimated.

Similar studies were performed in three patients during and after episodes of salt and water depletion. Two of the three, G.J. and R.P., have been mentioned in an earlier part of the paper. The third patient, C.C., was admitted for a study of the effects of sodium restriction on ileostomy excretion of electrolytes.

\section{RESULTS}

VOLUMES OF ILEOSTOMY MATERIAL AND URINE The volume of ileostomy material ranged from 380 to $1,500 \mathrm{ml}$./day with a mean volume of $690 \mathrm{ml}$./day. Repeated observations on a number of patients do not show any marked variation in daily volume. These volumes may therefore be regarded as representative of daily losses. Three of the group had an ileostomy volume greater than 1 litre per day and subsequently three-day faecal fat estimations were carried out. Abnormal faecal fat excretion of $26 \mathrm{~g}$./day was found in one patient. No similar studies are available for comparison but Smiddy, Gregory, Smith, and Goligher (1960) have stated that the volume from an established ileostomy is 200 to $500 \mathrm{ml} . /$ day.

The urine volumes in the ileostomy group ranged from 390 to $2,900 \mathrm{ml}$./day, with a mean of $970 \mathrm{ml}$.; seven had urine volumes less than $600 \mathrm{ml}$./day. When volumes less than $600 \mathrm{ml}$./day were found the collections were repeated to check their accuracy. The urine volume of the controls ranged from 750 to $2,130 \mathrm{ml}$./day with a mean of $1,410 \mathrm{ml}$./day. The difference in the means is significant at the 0.02

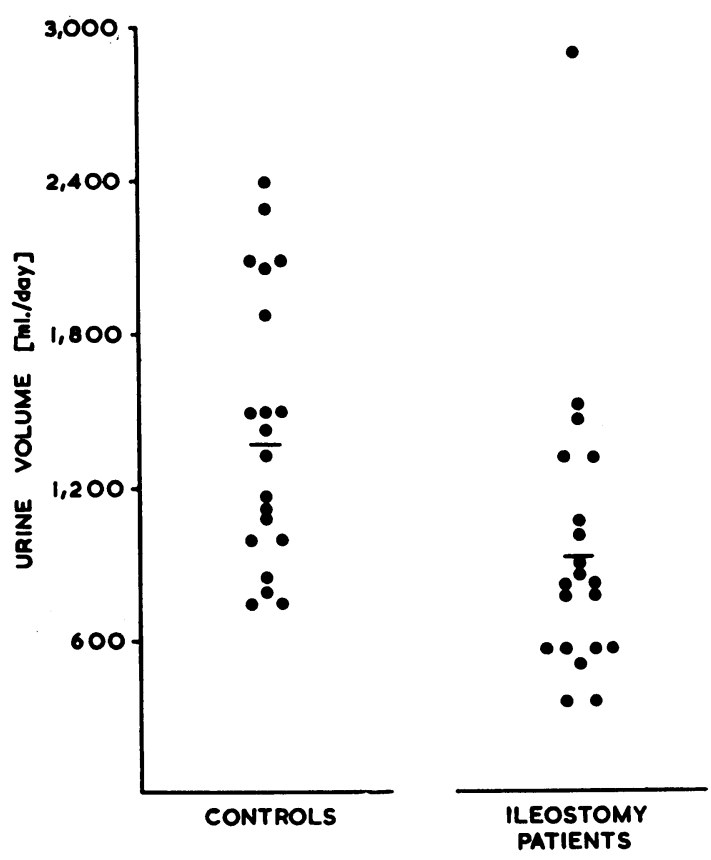

FIG. 1. Urine volume in controls and ileostomy patients.

level. Daily urine losses in both groups are shown in Fig. 1.

SODIUM EXCRETION IN ILEOSTOMY MATERIAL AND URINE The sodium loss in ileostomy material ranged from 44 to $197 \mathrm{mEq}$./day with a mean of $85 \mathrm{mEq}$./day. Sodium loss was always greater than the sodium loss in normal stools of 0.5 to $5 \mathrm{mEq}$./ day (Berger, 1960).

The sodium loss in the urine of the ileostomy group ranged from 1 to $250 \mathrm{mEq}$./day, the mean being $70 \mathrm{mEq}$./day. Marked differences were observed in the controls as urinary sodium loss ranged from 97 to $376 \mathrm{mEq}$./day, with a mean value of $180 \mathrm{mEq}$./day. The difference in urine sodium excretion in the two groups is highly significant $(P=0.001)$. Urinary sodium losses in the two groups are compared in Fig. 2. Fifteen members of the ileostomy group excreted less sodium in the urine than the smallest value of the control group of $97 \mathrm{mEq}$./day. Five of these 15 patients had urinary sodium losses of less than $10 \mathrm{mEq}$./day.

POTASSIUM EXCRETION IN ILEOSTOMY MATERIAL AND URINE Ileostomy material contained 2 to $12 \mathrm{mEq}$./ day of potassium, the mean being $5 \mathrm{mEq}$./day. Thus the ileostomy loss of potassium is within the range of the potassium loss in normal stools of 5 to 15 mEq./day (Berger, 1960). 


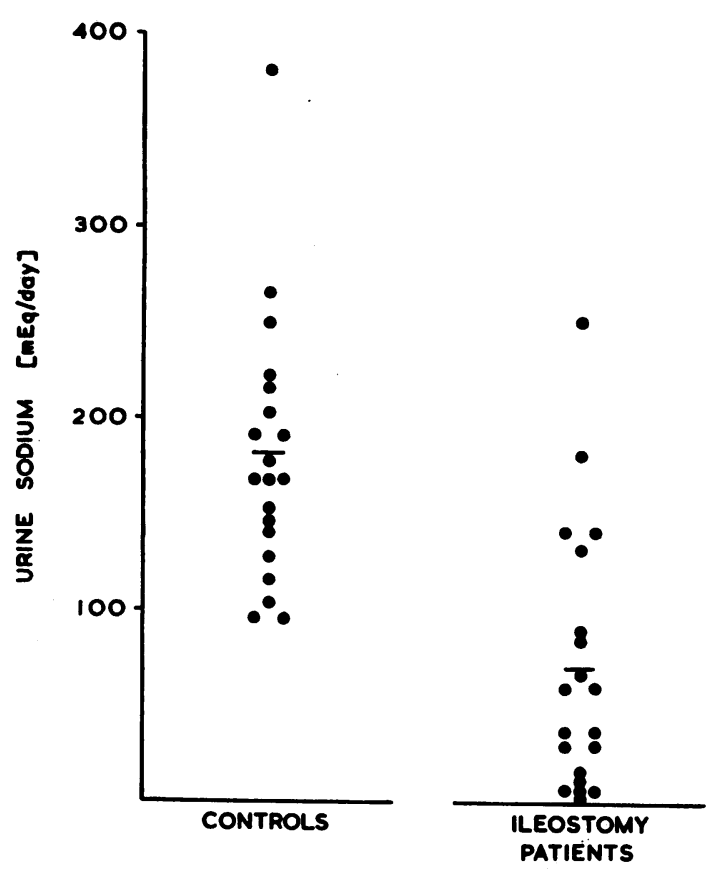

FIG. 2. Urinary sodium excretion in controls and ileostomy patients.

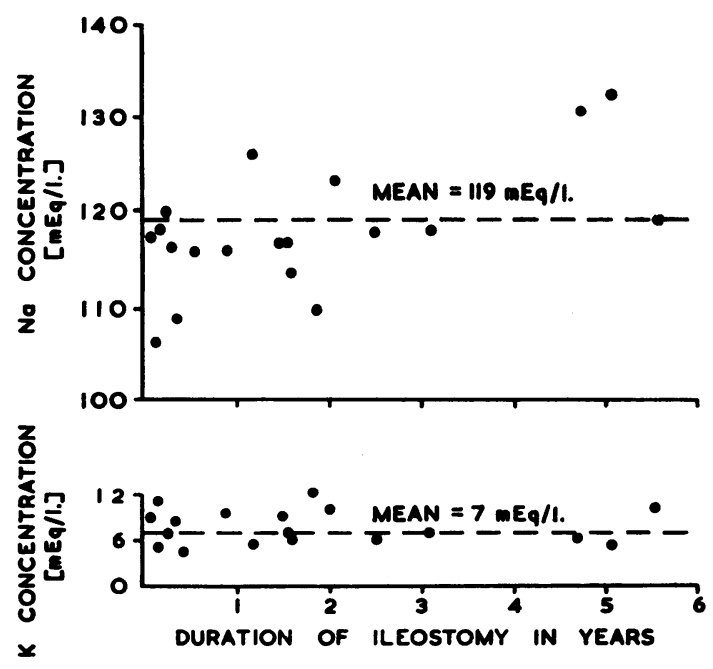

FIG. 3. The effect of time on the sodium and potassium concentrations of ileostomy material.

4
Urinary potassium loss in the ileostomy group ranged from 28 to $90 \mathrm{mEq}$./day with a mean of $62 \mathrm{mEq}$./day. Urinary potassium loss in the control group ranged from 52 to $134 \mathrm{mEq}$./day with a mean potassium loss of $78 \mathrm{mEq}$./day. The difference in the means was not significant. It was noted that several of the ileostomy patients with low urine volumes and low urine sodium levels had low urinary potassium excretion. The possible significance of this finding is being investigated.

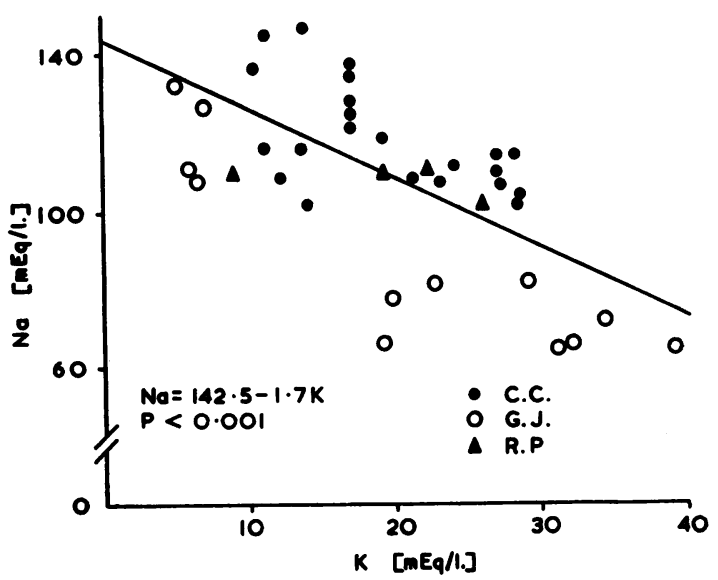

FIG. 4. Relationship between sodium and potassium concentration of ileostomy material during and after episodes of salt depletion.

CONCENTRATION OF SODIUM AND POTASSIUM IN ILEOSTOMY MATERIAL The sodium concentration ranged from 107 to $133 \mathrm{mEq}$./1. with a mean of $119 \mathrm{mEq} . / 1$. The potassium concentration ranged from 4 to $11 \mathrm{mEq}$. $/ 1$. with a mean of $7 \mathrm{mEq}$./1. There was no change in sodium and potassium concentration of ileostomy material with time (Fig. 3).

During episodes of sodium depletion in three patients, C.C., G.J., and R.P., the sodium concentration in ileostomy material always decreased and the potassium concentration increased. In one of the three, G.J., the sodium concentration fell to $60 \mathrm{mEq}$./1. and the potassium concentration rose to $40 \mathrm{mEq}$./l. Similar but less marked changes occurred in the other two patients. When the patients recovered the concentrations returned to their original levels. A negative regression was obtained when the sodium and potassium concentrations obtained during and after episodes of salt depletion were compared (Fig. 4). The relationship, $\mathrm{Na}=142.5-1.7 \mathrm{~K}$, was highly significant $(P<0.001)$. 


\section{DISCUSSION}

The majority of patients who leave hospital with an ileostomy enjoy good health. This is most obvious in patients who are able to take part in activities such as skiing, surfing, and golfing. However, an emphasis on the satisfactory adjustment of ileostomy patients to their new life has tended to obscure the fact that an increased daily loss of fluid and electrolytes from the small intestine is always present. Indeed, it has been said that colectomy with ileostomy in ulcerative colitis prevents further blood loss but does little to alter the loss of electrolytes (MacFadyen, Akre, Duncan, Flesch, and Mauser, 1954). Improvements in the techniques of fashioning an ileostomy and a more accurate replacement of body fluid and electrolytes in the post-operative period, when complications are most likely to develop, must surely have modified this opinion. Nevertheless, the increased fluid and electrolyte loss of ileostomy patients may still be responsible for the development of complications such as salt and water depletion as our experience shows. Other workers (Falconer, 1960) have had a similar experience.

Marked alterations in intestinal and renal losses of fluid and electrolytes were revealed by a study of apparently healthy ileostomy patients. Ileostomy sodium loss in each patient was found to be far greater than the sodium loss in normal stools. The effects of the increased sodium loss were frequently reflected in decreased urinary sodium excretion, an observation made originally by Welch, Wakefield, and Adams (1936). The reduction in urinary sodium excretion was most marked in five of our patients who were excreting less than $10 \mathrm{mEq}$./day. Fowler et al. (1959) have also noted an ileostomy patient in apparent good health despite the virtual absence of urinary sodium. Urinary sodium excretion was not always markedly reduced in patients with large ileostomy losses, indicating that low salt intakes were also responsible for the reduced urinary sodium excretion in some patients. Urine volumes in 10 of the ileostomy group were found to be smaller than the lowest volume found in the control group. The reduction was most marked in seven patients with urine volumes less than $600 \mathrm{ml}$./day. Small urine volumes were usually found in patients with large ileostomy volumes who also had reduced urinary sodium excretion. No differences were found in potassium excretion from the kidneys or intestine of ileostomy and control groups.

Factors regulating fluid and electrolyte excretion in the group of ileostomy patients have not been studied. High urine potassium-sodium ratios suggest that some ileostomy patients produce an increased amount of aldosterone.
The mean sodium and potassium concentrations of ileostomy material in healthy patients were 119 and $7 \mathrm{mEq}$./1. respectively. Our results are in close agreement with those of Smiddy et al. (1960) who studied patients in the post-operative period. They differ markedly, however, from those found by Lockwood and Randall (1949). The latter authors found a sodium concentration of $46 \mathrm{mEq}$./1. and a potassium concentration of $3 \mathrm{mEq} . / \mathrm{l}$. in material from an adapted ileostomy. We have not found any change in sodium and potassium concentration of ileostomy material obtained from patients who had had an ileostomy for periods ranging from one month to five years. This finding means that the amount of fluid and electrolyte loss from an ileostomy does not become smaller when normal ileostomy function begins. Thus the likelihood of patients developing salt and water depletion is always present as ileostomies are usually permanent.

The difficulties in obtaining ileal fluid from humans during salt depletion are overcome in patients with ileostomies. When salt depletion occurs we have been able to show that there is a decrease in the sodium concentration and an increase in the potassium concentration of ileostomy material. Moreover, Field, Dailey, Boyd, and Swell (1954) have found similar changes in ileal fluid from dogs. Increased potassium loss may then become a problem and lead in turn to potassium depletion. Analysis of the results we have obtained during salt depletion and following recovery indicate that there is a relationship between the concentration of sodium and potassium which can be expressed in the form $\mathrm{Na}=142.5-1.7 \mathrm{~K}$. One may speculate on the role of aldosterone in producing the altered concentration of these electrolytes when salt depletion exists but there is, as yet, no evidence of a direct effect on the intestine.

The findings of marked renal conservation of sodium and water in apparently healthy ileostomy patients explain why symptoms of salt and water depletion may readily develop during intercurrent illnesses. They have led to the following recommendations. Greater attention should be paid to salt and water needs, which may be assessed by measuring the sodium content and volume of a 24-hour collection of urine. Patients with low urine volumes should be advised to increase their fluid intake. Patients with a marked reduction in urinary sodium should be advised to eat more salt. All patients should be told of the necessity to increase salt and fluid intake, should diarrhoea or excessive sweating occur. They should also be told to seek medical advice if an illness is protracted or severe. Should salt depletion occur reduction in intestinal sodium loss will lead to increased intestinal loss of 
potassium, thereby increasing potassium requirements.

\section{SUMMARY}

The typical features of salt and water depletion have been noted in 10 out-patients with ileostomies. Complaints of headache, anorexia, nausea, muscle cramps, and convulsions were accompanied in some patients by oliguria and hypotension. Symptoms arose following episodes of gastroenteritis, intestinal obstruction, and excessive sweating.

Intestinal sodium and water loss was increased in each member of a group of 20 apparently healthy ileostomy patients. The mean ileostomy volume was $690 \mathrm{ml} . /$ day and its mean sodium content $95 \mathrm{mEq}$./ day. Increased losses and low intakes of sodium and water were frequently responsible for marked reduction in urinary sodium excretion and urine volume. Five patients excreted less than $10 \mathrm{mEq}$./day of sodium and seven had urine volumes less than $600 \mathrm{ml}$./day. Potassium losses in ileostomy material and urine were similar to those of normal individuals.

The mean sodium concentration of ileostomy material was $119 \mathrm{mEq} . / \mathrm{l}$. and the mean potassium concentration was $7 \mathrm{mEq}$./1. The electrolyte concentrations in ileostomy material were independent of the length of time the ileostomy had been present. Alterations in concentration occurred during episodes of salt depletion when the sodium concentration decreased and the potassium concentration increased.

Greater attention to the salt and water needs of apparently healthy ileostomy patients, particularly when marked renal conservation of sodium and water can be demonstrated, should reduce the incidence of salt and water depletion.

We wish to thank the members of the Ileostomy Association of N.S.W. for taking part in the study. The work was supported by the Bushell Trust and was assisted by a grant from the Post-Graduate Medical Foundation of the University of Sydney.

\section{REFERENCES}

Berger, E. Y. (1960). Intestinal absorption and secretion. In Mineral Metabolism. ed. Comar, C. L., and Bronner, F., Vol. I, Part A, p. 264. Academic Press, New York and London.

Brooke, B. N. (1956). Outcome of surgery for ulcerative colitis. Lancet, 2, 532-536.

Falconer, C. W. A. (1960). Ulcerative colitis. J. roy. Coll. Surg. Edinb., 5, 269-286.

Field, H., Dailey, R. E., Boyd, R. S., and Swell, L. (1954). Effect of restriction of dietary sodium on electrolyte composition of the contents of the terminal ileum. Amer. J. Physiol., 179, 477-480.

Fowler, D. I., Cooke, W. T., Brooke, B. N., and Cox, E. V. (1959). Ileostomy and electrolyte excretion. Amer. J. dig. Dis., n.s. 4, 710-720.

Lockwood, J. S., and Randall, H. T. (1949). The place of electrolyte studies in surgical patients. Bull. N.Y. Acad. Med., 25, 228-243.

MacFadyen, D. A., Akre, O. H., Duncan, J., Flesch, F, and Mauser, M. (1954). Electrolyte metabolism in ulcerative colitis patients before and after surgery. Gastroenterology, 27, 544-564.

Rogers, A. G., Bargen, J. A., and Black, B. M. (1954). Chronic ulcerative colitis: early and late experiences of 124 patients with ileac stomas. Ibid., 27, 383-393.

Smiddy, F. G., Gregory, S. D., Smith, I. B., and Goligher, J. C. (1960). Faecal loss of fluid, electrolytes, and nitrogen in colitis before and after ileostomy. Lancet, 1, 14-19.

Welch, C. S., Wakefield, E. G., and Adams, M. (1936). Function of the large intestine of man in absorption and excretion. Arch, intern. Med., 58, 1095-1110. 